\title{
Enhancing trappable antiproton populations through deceleration and frictional cooling
}

\author{
M. Zolotorev, A. Sessler, and G. Penn* \\ Center for Beam Physics, Lawrence Berkeley National Laboratory, Berkeley, California 94720, USA
}

J. S. Wurtele ${ }^{\dagger}$ and A. E. Charman

Department of Physics, University of California at Berkeley, Berkeley, California 94720, USA

(Received 5 December 2011; published 20 March 2012)

\begin{abstract}
CERN currently delivers antiprotons for trapping experiments with the antiproton decelerator (AD), which slows the antiprotons down to about $5 \mathrm{MeV}$. This energy is currently too high for direct trapping, and thick foils are used to slow down the beam to energies which can be trapped. To allow further deceleration to $\sim 100 \mathrm{keV}, \mathrm{CERN}$ is initiating the construction of ELENA, consisting of a ring which will combine rf deceleration and electron cooling capabilities. We describe a simple frictional cooling scheme that can serve to provide significantly improved trapping efficiency, either directly from the AD or first using a standard deceleration mechanism (induction linac or rf quadrupole). This scheme could be implemented in a short time. The device itself is short in length, uses accessible voltages, and at reasonable cost could serve in the interim before ELENA becomes operational, or possibly in lieu of ELENA for some experiments. Simple theory and simulations provide a preliminary assessment of the concept and its strengths and limitations, and highlight important areas for experimental studies, in particular to pin down the level of multiple scattering for low-energy antiprotons. We show that the frictional cooling scheme can provide a similar energy spectrum to that of ELENA, but with higher transverse emittances.
\end{abstract}

DOI: 10.1103/PhysRevSTAB.15.033502

PACS numbers: 37.10.Rs, 11.80.La, 34.50.Bw, 14.20.-c

\section{INTRODUCTION}

Scientific demand for low-energy antiprotons continues to grow for various experimental initiatives, including direct measurements of charge-to-mass ratios and production and trapping of antihydrogen, and eventually may lead to measurements of trapped neutral antimatter to test directly the weak equivalence principle and $C P T$ invariance [1-3].

The primary source of low-energy antiprotons remains the antiproton decelerator (AD) at CERN, but experiments typically suffer from low capture efficiency, because the antiprotons still exit the $\mathrm{AD}$ at average energies (around $5.3 \mathrm{MeV}$ ) far above achievable electrostatic trap depths. In order to trap the antiprotons, the beam is first sent through a degrading foil which slows the particles on average but leads to large particle losses and energy spread due to straggling effects, so only a small fraction of the antiproton source can typically be trapped.

To improve trapping efficiencies, the extra low-energy antiproton (ELENA) upgrade [4,5] to the AD is under development, which will use a postdecelerator and

\footnotetext{
*GEPenn@lbl.gov

${ }^{\dagger}$ Also at Center for Beam Physics, Lawrence Berkeley National Laboratory, Berkeley, CA 94720.

Published by the American Physical Society under the terms of the Creative Commons Attribution 3.0 License. Further distribution of this work must maintain attribution to the author(s) and the published article's title, journal citation, and DOI.
}

ring-based electron cooling to provide a source of $100 \mathrm{keV}$ antiprotons while maintaining high phase-space density. Other laboratories are also proposing low-energy antiproton deceleration and cooling rings, such as the facility for antiproton and ion research (FLAIR) [6] at GSI, but the time scale has been pushed back many years due to financial constraints.

Here we propose a simple scheme for longitudinal slowing and cooling of the antiproton beam delivered by the $\mathrm{AD}$, utilizing an optional deceleration section, based on either an induction linac or rf quadrupole (RFQ), followed by a degrading foil and finally a frictional cooling section. The frictional cooling stage consists of a series of thin carbon foils separated by reaccelerating electrostatic gradients. Such a scheme would not be quite as effective as ELENA would be, but is an adequate and available option for antiproton experiments, at lower costs and with a smaller footprint. Longitudinal losses should be comparable to that of ELENA, but there will be significant transverse losses even with large solenoidal fields maintained for confining the beam.

After providing a brief overview of our cooling concept, we present preliminary theoretical and numerical results from a simple equilibrium theory and Monte Carlo simulations, suggesting that frictional cooling can enhance the population of trapped antiprotons by a factor of 10 or more. Potentially, almost a factor of 100 improvement can be achieved if the frictional cooling is augmented by first decelerating the beam. We conclude with a discussion of 
advantages and limitations of the scheme, and of future directions for study.

\section{OVERVIEW OF FRICTIONAL COOLING FOR ANTIPROTONS}

Frictional cooling has been proposed and studied theoretically $[7,8]$ and experimentally $[9,10]$ in the context of muons. For antiprotons, frictional cooling might be used to compensate for the large mismatch that exists between the average kinetic energy of the antiproton beam exciting the $\mathrm{AD}$ and the kinetic energy of particles that typically can be trapped-several $\mathrm{MeV}$ versus a few $\mathrm{keV}$. To both shift the average energy and compress the energy spread of the beam, the antiproton beam is passed through a series of thin damping foils separated by electrostatic potential differences that partially reaccelerate the beam, as shown schematically in Fig. 1. Up to kinetic energies of about $\sim 85 \mathrm{keV}$, energy loss for antiprotons in the foils increases with increasing energy, so this design causes particles to converge to an equilibrium energy, analogous to a terminal velocity for falling objects in air. Because the particles lose energy on average in the direction opposite their velocities, but are reaccelerated only in the longitudinal direction, some transverse cooling is achieved as well. Transverse angles reach an equilibrium on the order of a fraction of a radian, and solenoidal magnetic fields are used to minimize growth in transverse spot size. As an additional benefit, the frictional cooling foils can actually facilitate the injection of particles in a magnetic trap; because the cooling foils randomize the transverse motion and nullify the adiabatic invariance of the antiproton magnetic moment related to the Larmor orbit, the foils will tend to prevent losses through the magnetic mirroring effect if the foils are positioned throughout the region of strong longitudinal magnetic field gradients.

One limitation of frictional cooling is the energy acceptance of the scheme. The stopping power through the foils starts to decrease with kinetic energy above $\sim 85 \mathrm{keV}$, and eventually drops below the value at the equilibrium energy. Typically, this occurs at $\sim 400 \mathrm{keV}$ kinetic energy. Particles above this energy get accelerated by the frictional cooling section, and so are not cooled. However, in practice the number of foils employed will be insufficient to capture this entire range of energies. This is why an initial degrading foil [11] must still be used, whose thickness is comparable to the range of the incident antiprotons at their mean energy, and which will therefore slow antiprotons to much lower energies. However, if the incident antiprotons have an energy of $\sim 5 \mathrm{MeV}$, straggling effects, or fluctuations in the energy loss, will lead to a broad initial energy distribution with a small fraction in the acceptance range for electrostatic trapping. The frictional cooling section is then designed to enhance the number of antiprotons with energies up to a few $\mathrm{keV}$, which typical cryogenic electrostatic traps are able to confine.

Cooling performance through frictional cooling is further limited by straggling, by surface roughness or inhomogeneity in the foils, and by the spread of angles in the beam driven by multiple scattering, which tend to counteract the cooling. Although the cooling effect applies to transverse angles as well as energy spreads, multiple scattering will lead to a residual angular spread which actually worsens at lower energies. To help control the effects of

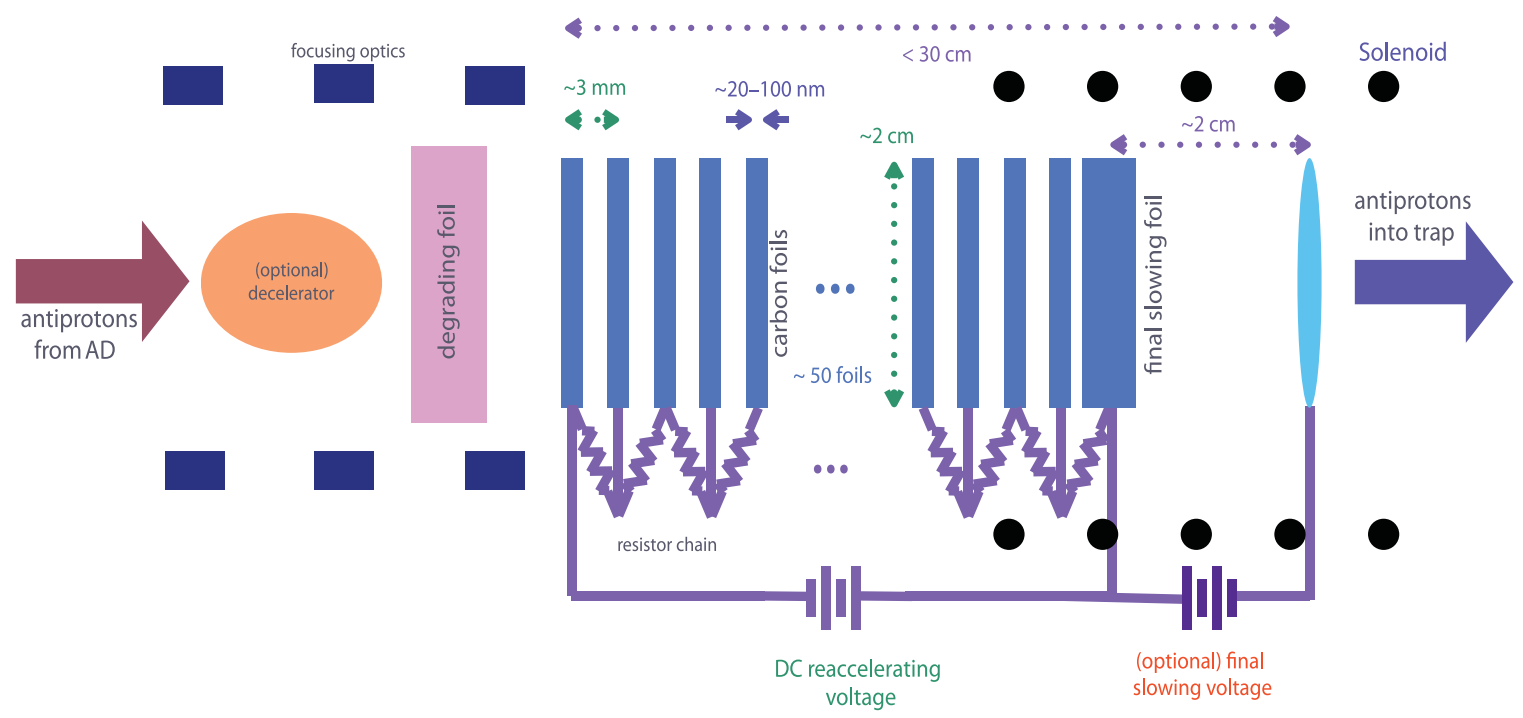

FIG. 1. Schematic of the proposed frictional cooling system. The diagram is not drawn to scale, but is intended to illustrate the major components. Cases with and without an additional active deceleration stage between the AD and the degrading foil are considered. Because the antiproton current is small and therefore the power requirements modest, presumably a resistor chain could be used to divide a single high voltage source. Final slowing might be achieved either by an oppositely polarized DC voltage or, as considered here, a thicker final foil. 
multiple scattering in the foils, we focus on low- $Z$ solid materials, particularly carbon, and the equilibrium energy of the beam is chosen to be somewhat higher than the typical acceptance of traps. In order to compensate for this, the very last foil will be thicker than the others in order to optimize the trapping efficiency. (An additional option, not utilized here, is to add a decelerating DC voltage between the cooling section and the trap.) The beam will also suffer some particle losses due to stopping or high-angle scattering in the foils. The number of foils, their thickness, and the potential differences between them are chosen to effect the desired cooling using currently available thin-foil technologies without prohibitive particle losses while keeping the overall length of device and total voltage drop within reasonable bounds.

\section{PHENOMENOLOGICAL BACKGROUND}

Next we review slowing, straggling, and angular scattering effects for antiprotons in thin solid foils, and infer phenomenological values for various approximate and parametrized expressions describing these dynamics. These considerations are employed in our equilibrium predictions and simulations.

Stopping power (in actuality, a force, not a power) is characterized by the average damping force $\langle d E / d s\rangle$, or loss of kinetic energy $E$ per unit path length $s$ in the material. At low velocities this is mostly due to inelastic collisions between the incident particles and target atoms, resulting in ionization and atomic excitation. The force is maximized at velocities of around $\alpha c$, where $c$ is the vacuum speed of light, and where $\alpha=e^{2} / \hbar c \approx 1 / 137$ is the fine structure constant. The important physics for frictional cooling occurs at velocities well below $\alpha c$, where the energy loss per distance is approximately proportional to the velocity, so that the incident particles experience something close to a linear friction force, like viscous drag. At extremely low velocities (below about $1 \mathrm{keV}$ kinetic energy for a proton or antiproton), the stopping power may plateau, as a roughly constant rate of loss due to elastic scattering off atoms may predominate.

Protons and antiprotons have very similar energy loss at high energies, but at nonrelativistic velocities antiprotons experience a somewhat smaller damping force [12-16] due primarily to differences in material polarization and exchange effects. This is known as the Barkas effect, which was first observed for pions but applies generally.

Recently, data on stopping powers for protons and antiprotons traveling through solid foils have been measured for light, moderate, and heavy elements, namely carbon $(Z=6)$, aluminum $(Z=13)$, nickel $(Z=28)$, and gold $(Z=79)$ [16], and have been shown to be in good agreement with a binary collision theory of Sigmund and Schinner [17-20].

Theories and experiments [16] confirm that at low velocities, stopping power is approximately proportional to the particle velocity. A plot of the stopping data in carbon foils is reproduced in Fig. 2. Restricting attention to solid carbon, their least-squares fit yields $\langle-d E / d s\rangle \propto$ $\beta^{0.96 \pm 0.14}$, and we can somewhat roughly approximate the stopping power in carbon at low energies $(<60 \mathrm{keV})$ as

$$
\left\langle-\frac{d E}{d s}\right\rangle \equiv \mathcal{S}(E) \approx \epsilon_{E} \frac{\rho}{\rho_{0}} \sqrt{E},
$$

where $\epsilon_{E} \approx 0.9 \mathrm{eV}^{1 / 2} / \mathrm{nm}$ for protons and $\boldsymbol{\epsilon}_{E} \approx$ $0.45 \mathrm{eV}^{1 / 2} / \mathrm{nm}$ for antiprotons, $\rho$ is the mass density, and $\rho_{0}=2.21 \mathrm{~g} / \mathrm{cm}^{2}$ is the nominal density for carbon.

Consistent with the fluctuation-dissipation theorems, the average stopping force is also associated with fluctuations in energy loss over a given distance characterized by a variance $\Omega^{2}$, which has two primary contributions adding in quadrature: from the stochastic nature of binary collisions, and from any surface roughness or density inhomogeneities in the actual target foil. The former contribution is often modeled using the well-known Bohr straggling value [21]:

$$
\Omega_{b}^{2}=4 \pi Z_{p}^{2} Z_{t}^{2} e^{4} n_{t} \Delta s,
$$

where $Z_{p} e$ is the charge of the incoming particles, $Z_{t}$ is the atomic number of the target species, $n_{T}$ is the number density of the target material, and $\Delta s$ is the path length through the target foil. However, straggling is also subject to a Barkas effect [22], with straggling of low-energy antiprotons typically reduced by up to a factor of 2 compared to protons at the same energy and in the same material. At low velocities, straggling is reduced from the Bohr values in a way which is not fully understood: an electron gas model implies $\Omega \propto \beta^{2}$ (where $\beta=|\mathbf{v}| / c$ is the normalized particle speed), while a binary collision model [18] predicts $\Omega \propto \beta^{6 / 5}$. Recent observations [22] are in reasonable agreement with the latter, with a

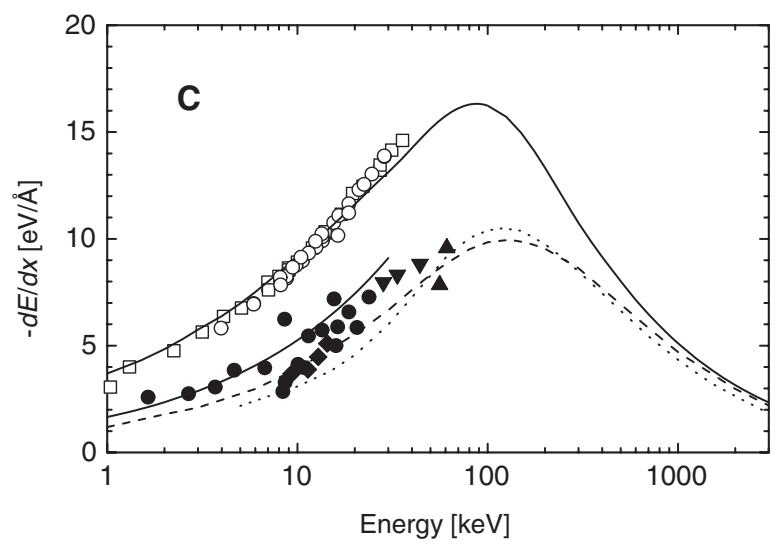

FIG. 2. Measured stopping power for protons (open symbols) and antiprotons (closed symbols) in solid carbon foils, reproduced from [16]. The lines show predictions of various theories. The Barkas effect is clearly evident. 
least-squares fit across several materials yielding an estimate of $\Omega \propto \beta^{0.9 \pm 0.2}$.

The inhomogeneous contributions can be expressed approximately in terms of the stopping power and thickness or density variations:

$$
\Omega_{\mathrm{inh}}^{2} \approx\left\langle\frac{d E}{d z}\right\rangle^{2}(\overline{\Delta s})^{2}\left[\frac{\left\langle\delta z^{2}\right\rangle}{\overline{\Delta s}^{2}}+\frac{\left\langle\delta \rho^{2}\right\rangle}{\rho^{2}}\right],
$$

where $\overline{\Delta s}$ is the nominal thickness of a target foil, $\delta z$ is deviation in thickness, or measure of surface roughness, and $\delta \rho=\rho-\langle\rho\rangle$ the local deviation in density. This contribution also leads to an approximately linear dependence of $\Omega$ on $\beta$ at low velocities.

Based on data for low-energy antiprotons in thin aluminum foils [22], reproduced in Fig. 3, we adopt an estimate for $\Omega$ that is linear in particle velocity (so that $\Omega^{2}$ is proportional to kinetic energy), specifically

$$
\frac{d \Omega^{2}}{d s} \approx \zeta_{E} \frac{\rho}{\rho_{0}} E
$$

where $\zeta_{E} \approx 0.17 \mathrm{eV} / \mathrm{nm}$ for protons, and $\zeta_{E} \approx$ $0.05 \mathrm{eV} / \mathrm{nm}$ for antiprotons. The Bohr straggling value corresponds in this case to $1.7 \times 10^{4} \mathrm{eV}^{2} / \mathrm{nm}$, which for protons matches the above expression at $E=100 \mathrm{keV}$.

Multiple scattering can be characterized by a differential cross section, or more simply for small-angle scattering, by the induced variance $\sigma_{\theta}^{2}$ in a single-plane divergence angle [23]. This variance will grow with the atomic numbers of the foil material or the incident particle, and with the foil thickness and number density, such that at low energies

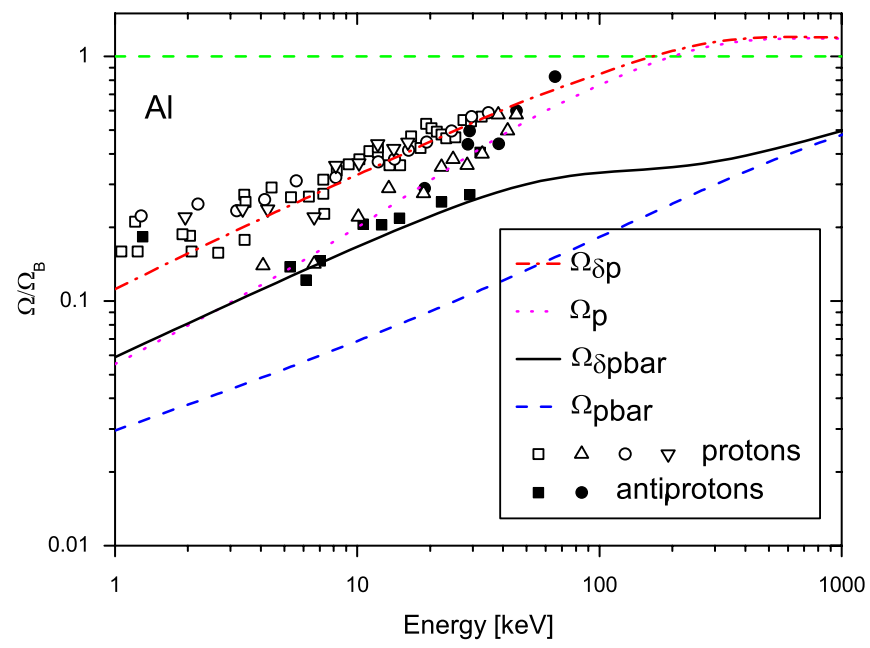

FIG. 3. Measured straggling normalized to Bohr straggling values for protons (open symbols) and antiprotons (closed symbols) in solid aluminum foils, reproduced from [22]. The solid line shows the predictions for antiprotons of a binary collision theory for a perfect foil, while the dashed line shows predictions based on a realistic level of foil inhomogeneities. The Barkas effect is apparent.

$$
\sigma_{\theta}^{2} \propto Z_{t}\left(Z_{t}+1\right) Z_{p}^{6} n_{t} \beta^{-4} \Delta s,
$$

plus some logarithmic corrections.

As a simple approximation describing the angular diffusion assuming small angles, an expression is commonly adopted based on a numerical fit to the Molière distribution, but with logarithmic corrections dropped:

$$
\frac{d \sigma_{\theta}^{2}}{d s} \approx \kappa_{\theta} \mu_{\theta} \frac{\rho}{\rho_{0}} E^{-2}
$$

where $\mu_{\theta} \approx 2.3 \times 10^{5} \mathrm{rad}^{2} \mathrm{eV}^{2} / \mathrm{nm}$ for protons in carbon. We have included an additional dimensionless "fudge factor" $\kappa_{\theta}$ for several reasons. While data [24] for multiple scattering of low-energy protons in carbon foils corroborate the energy dependence, the regime of interest here-particularly the thinness of the foils, low velocities, and large relative energy loss-do not match the usual application of this formula, and the extent of the Barkas effect is uncertain as well. We have considered values for $\kappa_{\theta}$ in the range $0.1<\kappa_{\theta} \leq 1.0$ for protons, and half those values for antiprotons, but hold out some hope that the actual value may be towards the low end of this region. Note that in the plot reproduced in Fig. 4, the ordinates are proportional to the standard deviation $\sigma_{\theta}$, not the variance $\sigma_{\theta}^{2}$, while the abscissa represents reciprocal kinetic energy $\left(E^{-1}\right)$, so the linear behavior seen therein is consistent with our assumed $E^{-2}$ energy for the growth of $\sigma_{\theta}^{2}$.

Because of the purely longitudinal reacceleration, frictional cooling also tends to damp angular deviations, countering multiple scattering. For small angles the angular compression within the electrostatic gradient is approximately

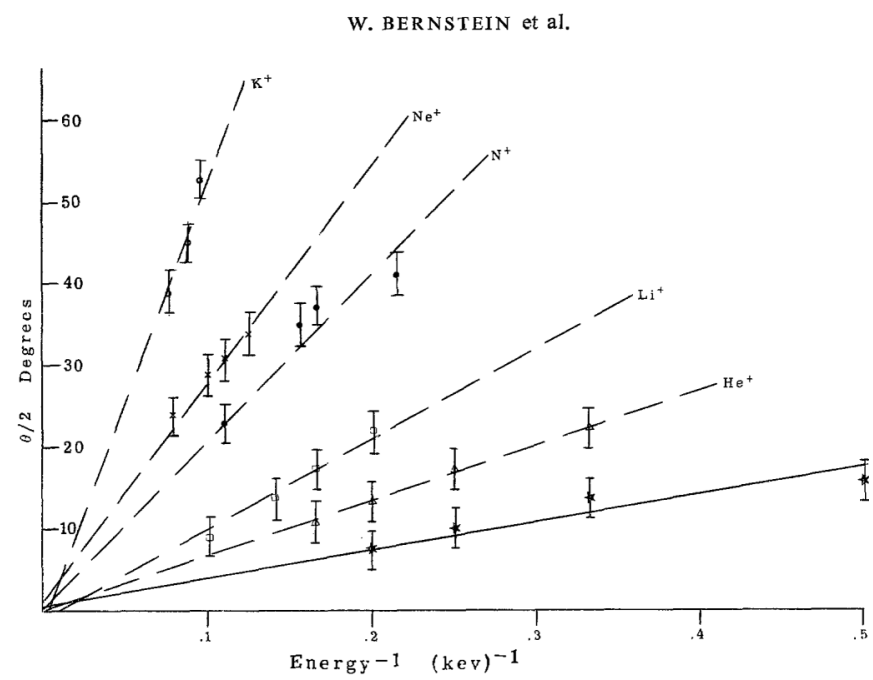

FIG. 4. Angular spread half-width due to multiple scattering (vertical axis) versus reciprocal incident energy in inverse $\mathrm{keV}$ (horizontal axis) for a variety of positive ions passing through carbon foils, reproduced from [24]. Linear least-squares fits are indicated. The bottom curve corresponds to protons $\left(H^{+}\right)$, and was used to estimate a value of $\kappa_{\theta} \approx 0.11$. 


$$
\frac{1}{\theta^{2}} \frac{d \theta^{2}}{d s} \approx-\frac{e \mathcal{E}_{z}}{E(z)},
$$

where $\mathcal{E}_{z}$ is the magnitude of the longitudinal electric field in the gap. For a particle to reach an equilibrium energy, this electric field must balance the energy loss at that energy. Since the angular scattering scales quadratically with $Z_{t}$, but the damping effect is proportional to the equilibrium energy loss which scales only linearly with $Z_{t}$, we are led to the use of low $-Z_{t}$ materials.

Annihilations are not expected to be significant, except for those particles that are essentially already stopped in the foils. Theoretical estimates $[25,26]$ predict the annihilation cross section to scale as $1 / \beta^{2}$; for $\sim 1 \mathrm{keV}$ antiproton, the mean-free path before annihilation corresponds to $\sim 100$ carbon foils, each of $\sim 100 \mathrm{~nm}$ thickness. So we anticipate that direct annihilation losses will be small even for low-energy particles while traveling through the complete cooling section.

\section{THEORETICAL ANALYSIS}

Transverse angles couple to the energy loss of particles through angle-dependent path length differences, with

$$
d s=d z / \cos [\theta(z)] \approx d z\left[1+\frac{1}{2} \theta(z)^{2}+\cdots\right],
$$

where $\theta(z)$ is the divergence angle of the particle trajectory at a longitudinal position $z$, and small angular deviations have been presumed. This suggests that with increasing angle, the equilibrium energy will have to decrease in order to accommodate the larger path length, just as thicker foils would cause a decrease in energy. This will also cause a correlation between energy and angle to develop.

Using the simple parametrized estimates for slowing, straggling, scattering, and energy/angle coupling described above, we can develop a simple expression predicting the equilibrium distributions of the low-energy antiprotons. Scattering angles are assumed small, and energy and angle fluctuations are assumed to be Gaussian. Interactions with matter and reaccelerating forces are treated as continuous, rather than lumped and alternating between foils and accelerating fields.

For a given equilibrium energy $\bar{E}$, we can estimate the equilibrium rms energy spread $\sigma_{E}$ to which the low-energy portion of the distribution function relaxes by finding the steady-state energy variance:

$\frac{d \sigma_{E}^{2}}{d z} \approx-2 \sigma_{E}^{2} \frac{d \mathcal{S}}{d E}+\frac{d \Omega^{2}}{d s}+\frac{1}{8}\left(\left\langle\theta_{x}^{2}+\theta_{y}^{2}\right\rangle\right)^{2} \frac{\mathcal{S}^{2}}{d \mathcal{S} / d E}=0$,

in which the first term represents the energy "squeezing" effects of the positive slope in stopping power, the second term represents the diffusive effects of energy straggling, and the last term roughly captures the effect of the angular spread in the beam due to correlations between angle and energy. The competition between multiple scattering and damping of angles should yield an equilibrium angular spread $\sigma_{\theta x}^{2}=\sigma_{\theta y}^{2} \approx\left(d \sigma_{\theta}^{2} / d s\right) \bar{E} / \mathcal{S}$. In the low-energy limit where $\mathcal{S} \propto E^{1 / 2}$, and when the angles are $\ll 1$, this can be rewritten as

$$
\frac{d \sigma_{E}^{2}}{d z} \approx-\sigma_{E}^{2} \frac{\mathcal{S}}{E}+\frac{d \Omega^{2}}{d s}+\left(\frac{d \sigma_{\theta}^{2}}{d s}\right)^{2} \frac{\bar{E}^{3}}{\mathcal{S}}=0 .
$$

The equilibrium value for the energy spread is given by

$$
\sigma_{E}^{2} \approx \frac{\bar{E}}{\mathcal{S}} \frac{d \Omega^{2}}{d s}+\frac{\bar{E}^{4}}{\mathcal{S}^{2}}\left(\frac{d \sigma_{\theta}^{2}}{d s}\right)^{2}
$$

where all energy-dependent quantities are to be evaluated at the equilibrium energy $\bar{E}$. When the estimated spread in angles approaches a radian, this expression significantly overestimates the energy spread contribution from multiple scattering.

A comparison between the predictions of this theory and Monte Carlo simulations as described below is shown in Fig. 5 for the case of $\kappa_{\theta}=0.05$. Here, the beam is assumed to start with a uniform energy distribution between 0 and $50 \mathrm{keV}$, and settings are adjusted so that, in equilibrium, antiprotons enter each foil at $5.7 \mathrm{keV}$ and exit at $5.0 \mathrm{keV}$. There are 150 foils, each $20 \mathrm{~nm}$ thick. The angular spread in this case satisfies $\sigma_{\theta x} \simeq 0.27$ radian, and the energy spread is $578 \mathrm{eV}$. The analytic values as defined above are $0.25 \mathrm{rad}$ and $574 \mathrm{eV}$. Inserting numbers, we observe that for equilibrium energies below $\sim 10 \mathrm{keV}$ and for $\kappa_{\theta}>0.1$, multiple scattering effects are more important than straggling in determining the achievable energy spread. When multiple scatter or particle losses dominate the dynamics, the distribution becomes lopsided with a tail at low energies as the particles at high angles drop in energy. As an

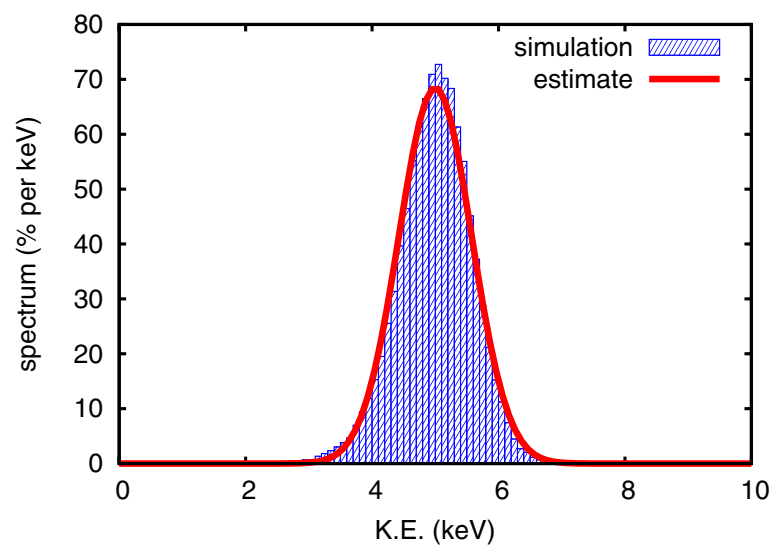

FIG. 5. Kinetic energy histogram for a broad energy distribution of antiprotons after a frictional cooling section with 150 carbon foils each $20 \mathrm{~nm}$ thick and a total potential of $109 \mathrm{kV}$. A value of $\kappa_{\theta}=0.05$ was used. The equilibrium energy is about $5 \mathrm{keV}$, with an rms energy spread of about $0.6 \mathrm{keV}$ and $<2 \%$ overall losses. For comparison we also show (solid line) the Gaussian fit based on a continuous medium analysis. 


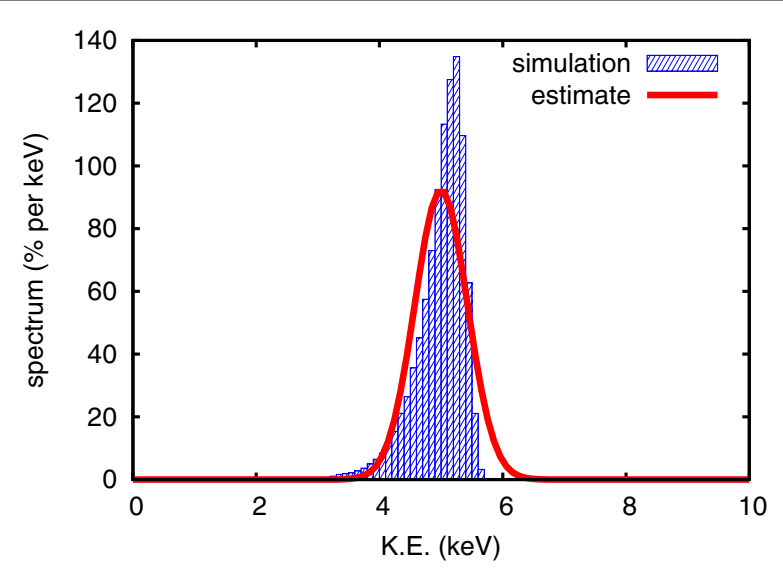

FIG. 6. Kinetic energy histogram for the same case as Fig. 5 but with energy straggling neglected. The equilibrium energy is about $5 \mathrm{keV}$, with an rms energy spread of about $0.4 \mathrm{keV}$ and $<2 \%$ overall losses. For comparison we also show (solid line) the Gaussian fit based on a continuous medium analysis.

example, if the physics of straggling is neglected in the above simulation, the distribution becomes that of Fig. 6. The simulated and analytic energy spreads in this case are 396 and $426 \mathrm{eV}$, respectively. Note that energy straggling does not have to be more important than multiple scatter in determining the equilibrium energy spread in order to cause the energy distribution to become approximately Gaussian. Alternatively, if not enough foils are used to reach equilibrium, or if the initial beam extends to very high energies, there will be an extended tail at high energies.

\section{DECELERATION SCHEMES}

In order to further increase the proportion of trappable antiprotons, between the $\mathrm{AD}$ and the frictional cooling section, we consider various ideas for deceleration, including the ELENA proposal, an induction linac, and a radiofrequency quadrupole (RFQ) system, and compare them to the use of only a foil. Every scheme, including ELENA, will also require some degrading foil after deceleration to further reduce energy before trapping. Note that the optimal choice for a degrading foil immediately preceding a trap may be different from that of a foil followed by a frictional cooling section. These deceleration options are fairly conventional and well studied. Thick foils used for deceleration are modeled using the similar physics as for frictional cooling, except that much higher energies must be included.

The AD [27] delivers about $2 \times 10^{7}$ antiprotons per bunch of about $0.3 \mu$ s duration at a mean kinetic energy of $5.3 \mathrm{MeV}$ every 1.8 minutes. The horizontal and vertical $(87 \%)$ emittances are about $1 \pi \mathrm{mmmrad}$ and $2 \pi \mathrm{mm}$ mrad, respectively, and the momentum spread after cooling and rebunching is about $0.1 \%$, corresponding to an rms kinetic energy spread of about $10.6 \mathrm{keV}$. Through electron cooling, ELENA can decelerate this beam to
$100 \mathrm{keV}$ with very small energy spread and low divergence. After the degrader, almost all of the beam lies within the energy acceptance of a typical trap. An induction linac can achieve a gradient of about $1 \mathrm{MeV} / \mathrm{m}$ for a long pulse, which can be increased somewhat if the pulse duration can be shortened from the nominal AD pulse length. The resulting energy spread is expected to be on the order of $25 \mathrm{keV}$ at $50 \mathrm{keV}$ energy. An RFQ can also slow the beam to $\sim 50 \mathrm{keV}$, with an even smaller energy spread. Note that one current experiment at CERN, ASACUSA, does employ an RFQ quadrupole system to decelerate bunches from the AD down to about $15 \mathrm{keV}$. But following a decelerator with active cooling can greatly enhance the number of low-energy antiprotons.

\section{NUMERICAL RESULTS}

In Monte Carlo simulations, we combined simple scaling laws for straggling and multiple scattering with interpolations based on tables of energy loss obtained from the TXPHYSICS software package [28], which uses SRIM [29,30] data. This is both to avoid any errors from oversimplifications in the above model used for our equilibrium estimate, and to be able to use a single calculation method for a range of kinetic energies from several $\mathrm{MeV}$ down to a fraction of a $\mathrm{keV}$ (which covers a wide range of different physical effects). Antiprotons are not included in these tables, and so the Barkas effect is separately estimated for the range of energies considered here. Annihilation effects for antiprotons were not included but are expected to be very small until the antiprotons are essentially stopped. The energy loss data is broadly consistent with Eq. (1), but the exponent for the energy dependence is closer to 0.4 for the energy range of interest, rather than 0.5 . In addition, a slightly more complicated form of straggling is considered than in Eq. (4), namely $d \Omega^{2} / d z=0.34(\mathrm{eV} / \mathrm{nm}) E /(E+$ $50 \mathrm{keV}$ ), which joins a linear slope at low energy to Bohr straggling at high energy. Straggling turns out to have a small effect on the frictional cooling section, although it is important for the degrading foil which slows the highenergy beam and thus affects the input into the frictional cooling stage. Both the degrader and frictional cooling foils are modeled with the same physics. Enhanced straggling due to surface roughness of the foils has not been modeled.

Following published experimental data, our nominal model for multiple scatter of low-energy protons has the form

$$
\begin{aligned}
\Delta \theta & =\frac{4.5 \mathrm{MeV}}{\beta c p} Z_{p}\left(\frac{x}{x_{0}}\right)^{1 / 2} \\
& \approx\left(0.11 \mu_{\theta} \frac{\rho}{\rho_{0}} \Delta z[\mathrm{~nm}]\right)^{1 / 2} \frac{1}{E[\mathrm{eV}]},
\end{aligned}
$$

where the second form applies in the limit of low kinetic energy and corresponds to the choice $\kappa_{\theta}=0.11$. Here $x$ is 
the mass-weighted thickness of the foil expressed in terms of $\mathrm{g} / \mathrm{cm}^{2}$, and $x_{0}$ is the radiation length in the same units, about $43 \mathrm{~g} / \mathrm{cm}^{2}$ for graphite. This is based on observations of the FWHM scattering angle passing through graphite foils of order $10 \mathrm{~nm}$ thickness, for protons at a several $\mathrm{keV}$ kinetic energy. However, this formula relies on the approximation whereby angles generated through multiple foils add in quadrature. We note that a commonly used formula [31],

$$
\Delta \theta=\frac{13.6 \mathrm{MeV}}{\beta c p} Z_{p}\left(\frac{x}{x_{0}}\right)^{1 / 2}\left(1+0.038 \ln \frac{x}{x_{0}}\right),
$$

yields a scattering angle that is very close to the earlier expression for a $10 \mathrm{~nm}$ foil when $x / x_{0} \approx 5 \times 10^{-8}$, yielding $4.9 \mathrm{MeV}$ rather than $4.5 \mathrm{MeV}$ for the prefactor in Eq. (12) when the average logarithmic dependence is absorbed into the coefficient, raising the effective $\kappa_{\theta}$ to 0.13 . Because the scattering profile is highly non-Gaussian in the tails of the distribution, there is some mismatch between a FWHM calculation and fit to a larger fraction of the beam. In addition the standard formula may not be suitable for the case when $\beta \ll \alpha$. These estimates need further experimental testing, especially against a configuration where a low-velocity proton or antiproton passes through many thin foils with reacceleration in between.

Antiprotons were assumed throughout to have energy loss, straggling, and multiple scattering [represented by $\mathcal{S}(E), \zeta_{E}$, and $\kappa_{\theta}$ ] all reduced by a factor of 2 compared to protons. The primary consequence of this Barkas effect is that foil thicknesses must be doubled for an antiproton cooling section as compared to a cooling section for protons, but the resulting performance will then be nearly identical. The exact details of how much multiple scattering, in particular, actually differs between protons and antiprotons at low energies will have a substantial impact on performance and required voltage. For antiprotons, the values of $\kappa_{\theta}$ examined here ranged from 0.05 to 0.4 .

For these simulations, the distribution of scattering angles was approximated as Gaussian, which forces the model to have the general form of Eq. (13) rather than Eq. (12). Comparing the two expressions requires careful consideration of the effective foil thickness, which is complex in the case of multiple foils alternating with reacceleration. If, in Eq. (13), we choose a thickness of $\sim 200 \mathrm{~nm}$, where the accumulated energy loss is a significant fraction of the nominal energy, that is equivalent to replacing the leading coefficient (4.5 MeV) in Eq. (12) with 6.1 MeV, and setting $\kappa_{\theta}=0.2$ for protons. A conservative

TABLE I. Numerical results of antiprotons cooling using different configurations and for different assumed values for multiple scattering strengths. The AD, induction linac, and RFQ schemes follow the indicated system with a degrading foil and possibly a frictional cooling section; the ELENA scheme is followed by a degrading foil only.

\begin{tabular}{|c|c|c|c|c|c|c|}
\hline Scheme & Initial $E$ & Initial $\sigma_{E}$ & $\kappa_{\theta}$ & Voltage & \# of cooling foils & $\%$ accepted \\
\hline \multirow[t]{6}{*}{$\mathrm{AD}$} & $5 \mathrm{MeV}$ & $10 \mathrm{keV}$ & 0.05 & 0 & 0 & 1.2 \\
\hline & & & 0.10 & 0 & 0 & 1.1 \\
\hline & & & 0.05 & $110 \mathrm{kV}$ & 35 & 13 \\
\hline & & & 0.10 & $122 \mathrm{kV}$ & 35 & 10 \\
\hline & & & 0.20 & $139 \mathrm{kV}$ & 35 & 7.2 \\
\hline & & & 0.40 & $152 \mathrm{kV}$ & 35 & 4.4 \\
\hline \multirow{6}{*}{ Induction linac } & $50 \mathrm{keV}$ & $25 \mathrm{keV}$ & 0.05 & 0 & 0 & 15 \\
\hline & & & 0.10 & 0 & 0 & 15 \\
\hline & & & 0.05 & $34 \mathrm{kV}$ & 12 & 57 \\
\hline & & & 0.10 & $50 \mathrm{kV}$ & 15 & 55 \\
\hline & & & 0.20 & $54 \mathrm{kV}$ & 15 & 41 \\
\hline & & & 0.40 & $50 \mathrm{kV}$ & 15 & 25 \\
\hline \multirow{6}{*}{ RFQ } & $50 \mathrm{keV}$ & $10 \mathrm{keV}$ & 0.05 & 0 & 0 & 37 \\
\hline & & & 0.10 & 0 & 0 & 35 \\
\hline & & & 0.05 & $34 \mathrm{kV}$ & 12 & 90 \\
\hline & & & 0.10 & $50 \mathrm{kV}$ & 15 & 85 \\
\hline & & & 0.20 & $54 \mathrm{kV}$ & 15 & 65 \\
\hline & & & 0.40 & $60 \mathrm{kV}$ & 15 & 40 \\
\hline \multirow[t]{4}{*}{ ELENA } & $100 \mathrm{keV}$ & $0.1 \mathrm{keV}$ & 0.05 & 0 & 0 & 91 \\
\hline & & & 0.10 & 0 & 0 & 85 \\
\hline & & & 0.20 & 0 & 0 & 73 \\
\hline & & & 0.40 & 0 & 0 & 59 \\
\hline
\end{tabular}


value for antiprotons might then be $\kappa_{\theta}=0.1$. As the angular scattering rate is increased, roughly similar trapping efficiencies can be achieved, but requiring more foils and increased total applied voltage across those foils. At the high end of values for scattering considered here, performance begins to degrade more significantly.

For most of the examples presented, two distinct configurations were considered, each separately optimized to maximize the number of antiprotons with longitudinal energy below a nominally trappable threshold of $3 \mathrm{keV}$ : one where the beam passes through a single degrader to slow the beam, and a second case where a thinner degrader is used followed by multiple stages of frictional cooling. The exceptions are for the current AD parameters, where the assumed degrader is the same thickness in either case, and for ELENA parameters, where even without frictional cooling almost all antiprotons can be slowed to below the $3 \mathrm{keV}$ limit without being stopped. The energy spread for ELENA is sufficiently low that the degrader foil could be replaced by purely electric deceleration for full longitudinal capture.

The geometry of the slowing/cooling section consists of a degrading foil to slow the incident beam, followed by multiple foils with a thickness of $100 \mathrm{~nm}(50 \mathrm{~nm}$ for protons), separated by $4 \mathrm{~mm}$ gaps with a voltage per gap in the range of 3-5 keV. The final foil can be made thicker to further slow the cooled antiprotons to below $3 \mathrm{keV}$ longitudinal energy. To reduce the required voltage and number of foils, the frictional cooling stage is sufficiently short and the voltage sufficiently low that the beam distribution has not equilibrated, and in fact the equilibrium energy would be near or below $3 \mathrm{keV}$. From the contribution to energy straggling of foil inhomogeneities as in Eq. (3), it is apparent that if the relative transverse inhomogeneities in each foil is above $\left(\zeta_{E} / \Delta s\right)^{1 / 2} / \epsilon_{E} \simeq$ $5 \%$, the effect of inhomogeneities alone rises above the assumed level of energy fluctuations. This corresponds to an effective roughness of $<5 \mathrm{~nm}$ for a $100-\mathrm{nm}$ foil. Inhomogeneities above this level would then act as an increase in straggling. In Ref. [22], fits to the data for aluminum and gold foils yield an effective thickness variation of 6 and $8 \mathrm{~nm}$, respectively.

As seen in Table I, the RFQ plus frictional cooling configuration can yield similar output to ELENA followed by a degrading foil, over a range of values of the scattering rate. At increasing values of $\kappa_{\theta}$, both more foils and higher voltage between foil are needed, requiring significantly more total voltage drop. The kinetic energy spectrum of different configurations assuming antiprotons scatter according to $\kappa_{\theta}=0.10$ is shown in Fig. 7. The
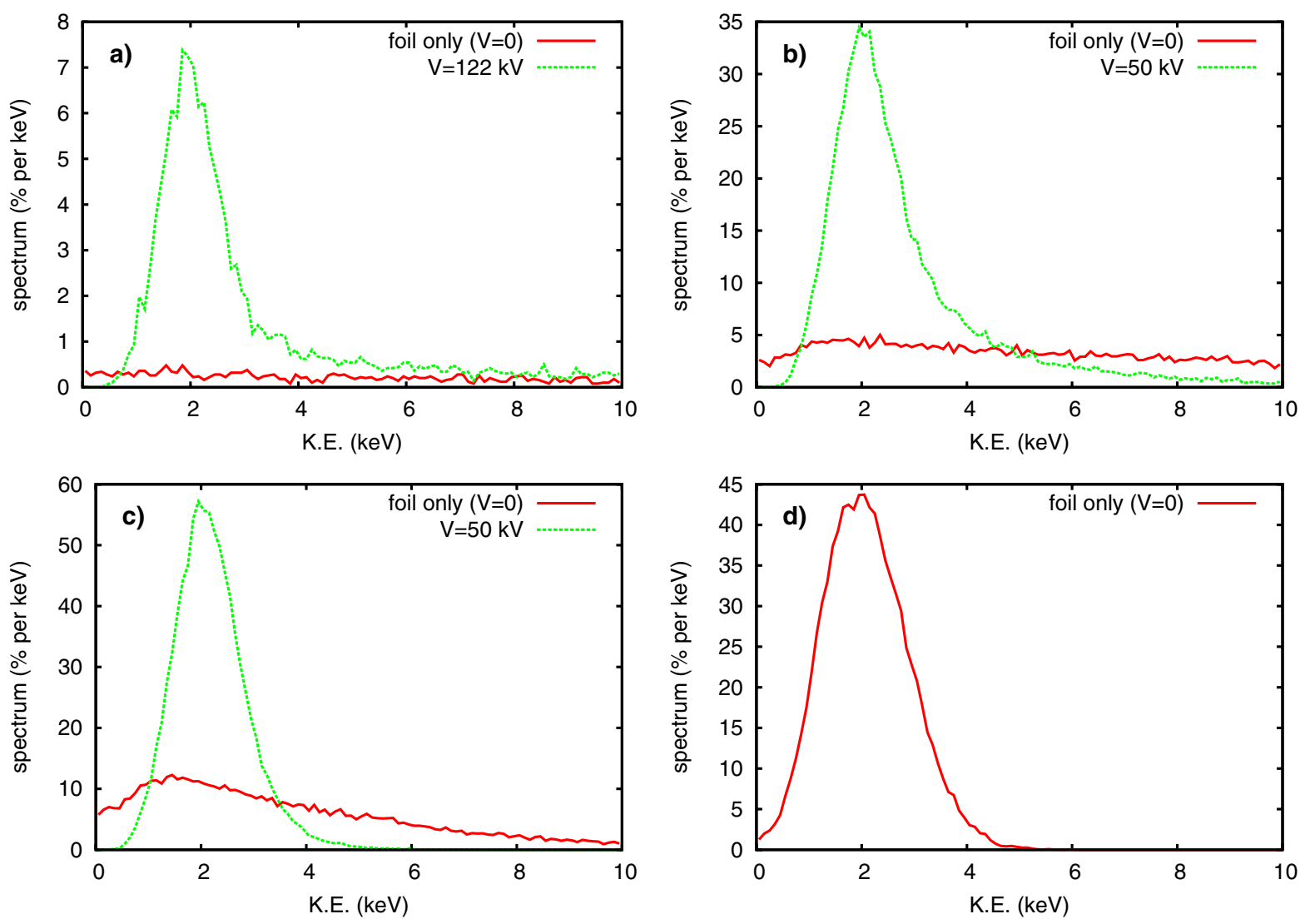

FIG. 7. Antiproton spectrum for $\kappa_{\theta}=0.1$ with or without frictional cooling, from the various configurations: (a) antiprotons straight from the $\mathrm{AD}$, or further deceleration from (b) induction linac, (c) RFQ, or (d) ELENA. 
scaling of some scenarios with scattering level is shown in Figs. 8-10. The frictional cooling parameters have been readjusted for each value of $\kappa_{\theta}$. These spectra are given in units of percentage of the original beam population per $\mathrm{keV}$, as a function of per-particle kinetic energy. Note that the spectrum is given in terms of total kinetic energy per

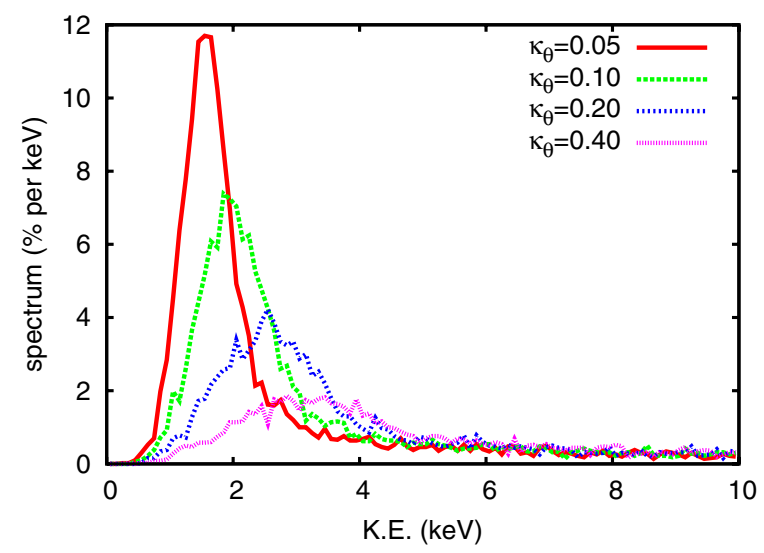

FIG. 8. Antiproton spectrum from the AD followed by degrader foil and frictional cooling section for various values of $\kappa_{\theta}$.

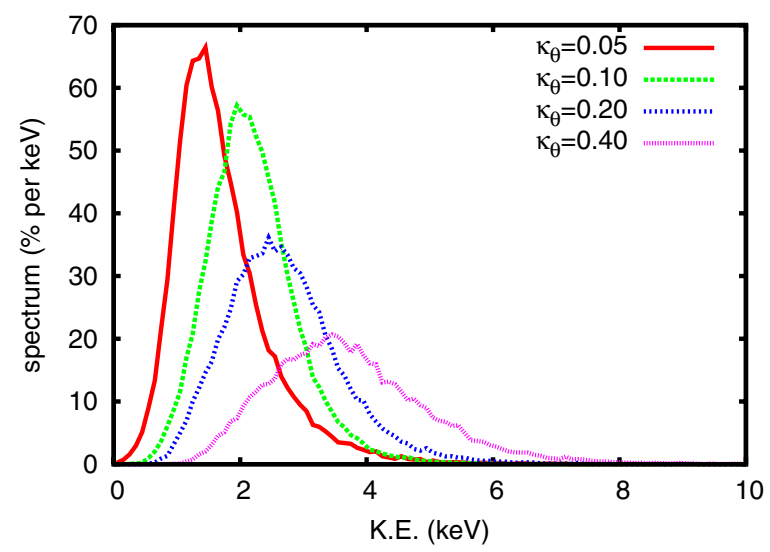

FIG. 9. Antiproton spectrum from RFQ followed by degrader foil and frictional cooling section for various values of $\kappa_{\theta}$.

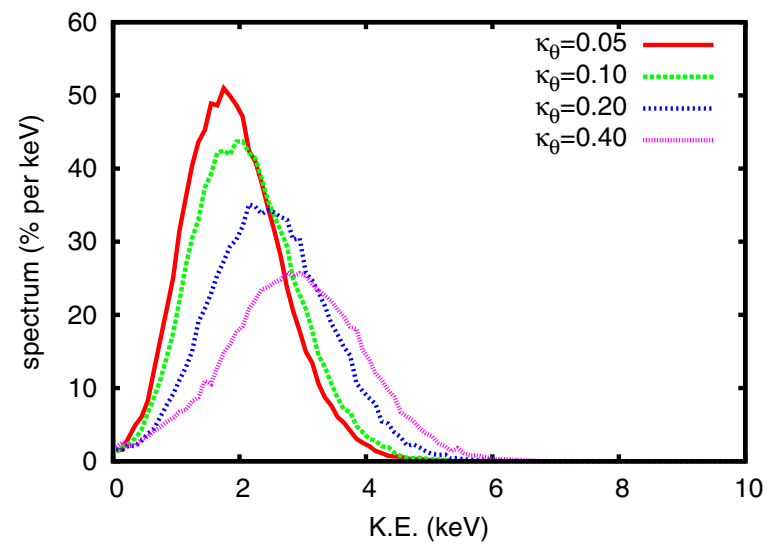

FIG. 10. Antiproton spectrum from ELENA followed by degrader foil for various values of $\kappa_{\theta}$. particle, while the acceptance criterion is ultimately based on longitudinal kinetic energy. More quantitative estimation of the performance requires knowledge of the transverse acceptance of the antiproton trapping and cooling. This requires detailed modeling of the trapping system, the magnetic fields, and the antiproton cooling process. However, the transverse phase-space profile of the beam after a frictional cooling stage should be comparable to or smaller than the profile after a single degrader foil, so estimates of relative performance gains should be fairly close. The improved transverse brightness resulting from ELENA may yield further gains that are not identified by the present study.

\section{DISCUSSION AND CONCLUSIONS}

Preliminary theory and simulations suggest that, as long as the multiple scattering cross section is sufficiently small, a simple frictional scheme applied to antiproton bunches delivered by the $\mathrm{AD}$ cooling can significantly enhance the numbers of trappable particles with few-keV kinetic energies, perhaps by an order of magnitude. At the expense of greater complexity, cost, and real estate, trapping should be enhanced considerably more by supplementing the degrading foil for the initial slowing of the antiprotons out of the $\mathrm{AD}$ with other methods such as an induction linac or RFQ, assuming that a beam with large divergence angles can be transported effectively to the trap.

When optimized for energy manipulation, frictional cooling can reduce down to few $\mathrm{keV}$ levels both the mean energy and energy spread of a subpopulation of the beam that is incident at sufficiently low energy (below several hundred $\mathrm{keV}$ if sufficient voltage is applied). It is important to note that the rms angular divergence may be increased significantly, the spot size moderately, and the bunch length somewhat, and the higher-energy portion of the beam will not be cooled. Both the theory and simulations are based on a number of simplifying assumptions that should be replaced by a more complete treatment, particularly the use of small-angle expansions. Better data on the extent of the Barkas effect for scattering in low-energy antiprotons would be particularly useful for more accurate simulations.

The requirements for a frictional cooling section seem technologically feasible: $\leq 50$ carbon foils, each about $100 \mathrm{~nm}$ thick, plus or minus a few percent inhomogeneity, and if necessary fitting into a compact space; a total DC voltage source of $\lesssim 150 \mathrm{kV}$; and an induction linac or RFQ unit to improve performance beyond what can be achieved by going from the AD directly into a degrading foil. Transverse inhomogeneities in foil thickness should ideally be below $5 \mathrm{~nm}$, which is about a factor of 2 less than previous estimates for metallic foils [22]. Otherwise, a higher effective level of energy straggling must be used in the calculations; this is only expected to impact the results in the case of the smallest level of multiple 
scattering considered $\left(\kappa_{\theta}=0.05\right)$. Variations of the average thickness among the individual foils are not a concern, as they can be corrected for by changing the voltage on individual gaps.

In addition, because in all cases studied the equilibrium divergence angles become moderately large, a strong solenoidal field may be needed, rising to $\sim 3 \mathrm{~T}$, in order to provide transverse confinement. In many trapping applications the frictional cooling section may be able to piggy-back on the existing solenoidal field, and if the foils are distributed throughout the region along the beam line where the longitudinal field gradient is large, they will actually tend to suppress losses due to magnetic mirroring by nullifying the related adiabatic invariant.

When using either a degrading foil or a decelerator, multiple scattering at the lowest energies is a determining factor for performance. Unfortunately, the rates of multiple scattering are rather poorly known for low-energy antiprotons in carbon or other solid materials. Observations suggest cross sections for low-energy protons are less than the Molière prediction, and the Barkas effect suggests antiproton scattering cross sections should be smaller still, but better understanding and measurement of these cross sections would be very useful in assessing the achievable efficacy of frictional cooling. Annihilation effects are expected to be small, but should perhaps be studied further. Also because of the Barkas effect, note that if such a scheme is to be tested using a proton beam, one must either use thinner foils or higher voltages between foils to see equivalent results.

The ELENA deceleration and cooling ring is predicted to be effective and robust. The performance is at least comparable to the scheme using an RFQ-based decelerator followed by frictional cooling, and will be further enhanced through transverse cooling, the effects of which have not been considered here. Using an induction linac may be simpler than an RFQ, but less effective. Without any additional active deceleration before cooling, the bulk of the antiproton beam cannot be trapped, but frictional cooling should still improve the trappable fraction by an order of magnitude or so compared to the use of a single degrading foil only. While frictional cooling offers a straightforward means to improving trapping efficiency, the current rapid timetable for ELENA may remove the incentive to pursue a frictional cooling stage at CERN.

When a degrading foil is used before the frictional cooling section proper, its thickness should be carefully chosen to maximize performance. In either the case of foils or linacs or both, more complete understanding of the phase-space acceptances of the downstream trap will be needed to optimize performance in specific applications. In addition, while we have considered for simplicity a repetition of identical foils and gap voltages, tapering of these quantities might further improve performance. Optimization and improved simulation of the cooling layout, as well as more realistic modeling of the upstream degrading foil and downstream trapping dynamics, needs to be done. More optimizations should also be considered, for example the potential between successive foils could be ramped to either reduce the required voltage or increase performance.

\section{ACKNOWLEDGMENTS}

The authors thank Joel Fajans and Niels Madsen for useful conversations and communications, as well as Alex Friedman and all of the members of the LBNL Ion Fusion Group, for helpful discussions concerning induction linacs. This work was supported by the Director, Office of Science, Office of HEP of the U.S. Department of Energy under Contracts No. DE-AC02-05CH11231, No. DE-FG02-06ER54904, and No. DE-FG0204ER41289.

[1] W. Oelert, Int. J. Mod. Phys. A 26, 390 (2011).

[2] E. Widmann, Acta Phys. Pol. B 41, 249 (2010) [http://thwww.if.uj.edu.pl/acta/vol41/abs/v41p0249.htm].

[3] N. Madsen, Phil. Trans. R. Soc. A 368, 3671 (2010).

[4] W. Oelert et al. (AD User Community), CERN Report No. CERN-SPSC-2009-026, 2009.

[5] P. Belochitskii, in Proceedings of COOL 2007, Bad Kreuznach, Germany, 2007, edited by R. W. Hasse and V.R.W. Schaa (Gesellschaft für Schwerionenforschung mbH, Darmstadt, Germany, 2007), p. 6-10 [http:// accelconf.web.cern.ch/AccelConf/c107/PAPERS/ MOM1I02.PDF].

[6] C. Welsch and J. Ullrich, Hyperfine Interact. 172, 71 (2006).

[7] R. Galea, A. Caldwell, and S. Schlenstedt, J. Phys. G 29, 1653 (2003).

[8] T. J. Roberts and D. M. Kaplan, in Proceedings of the 23rd Particle Accelerator Conference, Vancouver, Canada, 2009 (TRIUMF, Vancouver, 2009), pp. 2370-2372 [http://accelconf.web.cern.ch/AccelConf/PAC2009/papers/ we6pfp096.pdf].

[9] M. Mühlbauer, H. Daniel, F. J. Hartmann, P. Hauser, F. Kottmann, C. Petitjean, W. Schott, D. Taqqu, and P. Wojciechowski, Hyperfine Interact. 119, 305 (1999).

[10] R. Galea, Nucl. Phys. B149, 295 (2005).

[11] H. Kalinowsky, Hyperfine Interact. 76, 73 (1993).

[12] S. P. Møller, Nucl. Instrum. Methods Phys. Res., Sect. B 48, 1 (1990).

[13] R. Medenwaldt, S. P. Møller, E. Uggerhøj, T. Worm, P. Hvelplund, H. Knudsen, K. Elsener, and E. Morenzoni, Phys. Lett. A 155, 155 (1991).

[14] A. Adamo et al., Phys. Rev. A 47, 4517 (1993).

[15] G. Schiwietz, U. Wille, R. Díez Muiño, P. D. Fainstein, and P. L. Grande, J. Phys. B 29, 307 (1996).

[16] S. P. Møller, A. Csete, T. Ichioka, H. Knudsen, U. I. Uggerhøj, and H.H. Andersen, Phys. Rev. Lett. 88, 193201 (2002).

[17] P. Sigmund and A. Schinner, Eur. Phys. J. D 12, 425 (2000). 
[18] P. Sigmund and A. Schinner, Eur. Phys. J. D 15, 165 (2001).

[19] P. Sigmund and A. Schinner, Nucl. Instrum. Methods Phys. Res., Sect. B 193, 49 (2002).

[20] P. Sigmund and A. Schinner, Nucl. Instrum. Methods Phys. Res., Sect. B 212, 110 (2003).

[21] N. Bohr, Philos. Mag. Ser. 6 30, 581 (1915).

[22] S. P. Møller, A. Csete, T. Ichioka, H. Knudsen, H.-P. E. Kristiansen, U. I. Uggerhøj, H. H. Andersen, P. Sigmund, and A. Schinner, Eur. Phys. J. D 46, 89 (2008).

[23] C. Amsler et al. (Particle Data Group), Phys. Lett. B 667, 1 (2008).

[24] W. Bernstein, A. J. Cole, and R. L. Wax, Nucl. Instrum. Methods 90, 325 (1970).

[25] K. V. Protasov, G. Bonomi, E. Lodi Rizzini, and A. Zenoni, arXiv:nucl-th/9911005.

[26] C. J. Batty, E. Friedman, and A. Gal, Nucl. Phys. A689, 721 (2001).

[27] P. Belochitskii, J. Bosser, J. Buttkus, C. Carli, F. Caspers, V. Chohan, D. Cornuet, T. Eriksson, A. Findlay, M.
Giovannozzi, B. Holzer, R. Maccaferri, N. Madsen, M. Marchesotti, S. Maury, D. Mohl, S. Pasinelli, F. Pedersen, L. Soby, J. Tan, and G. Tranquille, in Proceedings of the 2001

Particle Accelerator Conference, Chicago, 2001, edited by P. Lucas and S. Webers (IEEE, New York, 2001), pp. 580-584 [http://accelconf.web.cern.ch/ AccelConf/p01/PAPERS/ROPA008.PDF].

[28] P. H. Stoltz, S. Veitzer, R. H. Cohen, A. W. Molvik, and J.-L. Vay, Nucl. Instrum. Methods Phys. Res., Sect. A 544, 502 (2005).

[29] J. F. Ziegler, J. P. Biersack, and U. Littmark, The Stopping and Range of Ions in Solids (Pergamon, New York, 1985). See also www.srim.org.

[30] J. F. Ziegler, Nucl. Instrum. Methods Phys. Res., Sect. B 219-220, 1027 (2004).

[31] G. R. Lynch and O. I. Dahl, Nucl. Instrum. Methods Phys. Res., Sect. B 58, 6 (1991). 